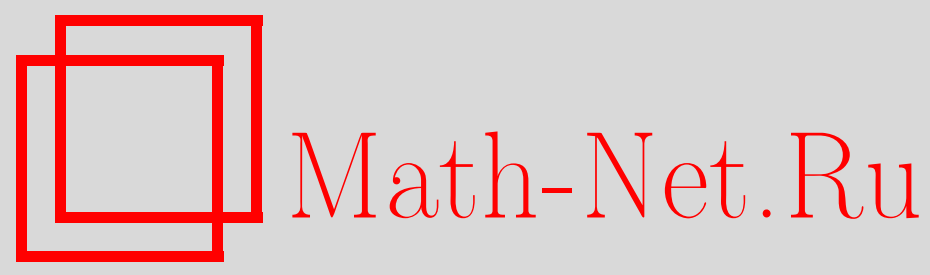

В. П. Маслов, Статистический ансамбль и квантование термодинамики, Матем. заметки, 2002, том 71, выпуск 4, 558 566

DOI: https://doi.org/10.4213/mzm367

Использование Общероссийского математического портала Math-Net.Ru подразумевает, что вы прочитали и согласны с пользовательским соглашением http://www . mathnet.ru/rus/agreement

Параметры загрузки:

IP : 54.197 .130 .99

26 апреля 2023 г., 17:50:04

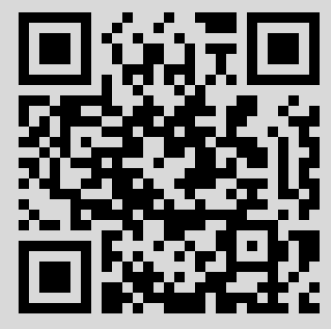




\section{СТАТИСТИЧЕСКИЙ АНСАМБЛЬ И КВАНТОВАНИЕ ТЕРМОДИНАМИКИ}

\section{В.П. Маслов}

В статье приведен вьвод уравнений для квантованной термодинамики исходя из ансамбля Гиббса, и получено такое представление этого нового уравнения, которое позволяет формально перейти к уравнениям БКШ.

Библиограбфия: 5 названий.

В предыдущих работах автора [1], [2] для идеального газа были введены операторы свободной энергии для разных случаев ультравторичного (по “холостым"; по "холостым" и "парам") и вторичного квантования. Операторы свободной энергии в [1], [2] были введены, исходя из тождеств для статистической суммы. Они зависели от “духов" параметров, от которых в термодинамическом пределе не зависела асимптотика минимального собственного значения оператора свободной энергии, причем эта асимптотика совпадает с асимптотикой статистичекой суммы идеального газа. В данной статье дается обобщение введенного в [1], [2] оператора свободной энергии и рассматриваются системы со взаимодействием. При этом для квантования свободной энергии используется фундаментальное представление о статистическом ансамбле [3].

Рассмотрим сначала идеальный газ. Будем считать, что $N$ частицнаходятся на трехмерном торе $\mathbb{T}$, все три окружности которого равны $L$. Пространство состояний такой квантовой системы есть $L_{2}\left(\mathbb{T}^{N}\right)$. Оператор Гамильтона идеального газа имеет вид

$$
\widehat{H}_{N}=-\frac{\hbar^{2}}{2 m} \sum_{j=1}^{N} \Delta_{j}
$$

где $\Delta_{j}=\sum_{\alpha=1}^{3} \partial^{2} / \partial x_{j \alpha}^{2}$ - оператор Лапласа, $x_{j \alpha}$ - координаты $j$-й частищы на торе $\mathbb{T}$, $x_{j}=\left(x_{j 1}, x_{j 2}, x_{j 3}\right)$. Стационарное уравнение Шрёдингера, отвечающее гамильтониану (1):

$$
\widehat{H}_{N} \Psi\left(x_{1}, \ldots, x_{N}\right)=E \Psi\left(x_{1}, \ldots, x_{N}\right),
$$

где $\Psi\left(x_{1}, \ldots, x_{N}\right)$ - функция из пространства $L_{2}\left(\mathbb{T}^{N}\right)$. Мы не считаем, что газ состоит из фермионов или бозонов, поэтому никаких условий симметрии или антисимметрии на функцию $\Psi$ не накладьвается (т.е. частицы различимы). Из решений уравнения (2) можно выбрать полную ортонормированную в $L_{2}\left(\mathbb{T}^{N}\right)$ систему функций

$$
\Psi_{\{K\}}\left(x_{1}, \ldots, x_{N}\right)=\frac{1}{L^{3 N / 2}} e^{i \sum_{j=1}^{N} K_{j} x_{j}},
$$


где $K_{j}$ - трехмерные векторы вида $K_{j}=\frac{2 \pi}{L}\left(l_{j 1}, l_{j 2}, l_{j 3}\right), l_{j \alpha}$ - целые числа, $K_{j} x_{j}=$ $\sum_{\alpha=1}^{3} K_{j \alpha} x_{j \alpha},\{K\}$ - обозначение для набора векторов $K_{1}, K_{2}, \ldots, K_{N}$. Соответствующее функции (3) собственное значение оператора (1) имеет вид

$$
E_{\{K\}}=\sum_{j=1}^{N} \frac{\hbar^{2} K_{j}^{2}}{2 m}
$$

Удельная свободная энергия $F(\theta, n)$ [3] системы различимых частиц с гамильтонианом $\widehat{H}_{N}$ при температуре $\theta$ и плотности $n$ определяется как термодинамический предел величины

$$
-\frac{\theta}{N} \ln \left(\frac{1}{N !} \operatorname{Sp} e^{-\widehat{H}_{N} / \theta}\right)=\frac{F\left(\theta, L^{3}, N\right)}{N} .
$$

Под термодинамическим пределомпонимается предел при $N \rightarrow \infty, L \rightarrow \infty, N / L^{3}=n$ - постоянная величина, $F\left(\theta, L^{3}, N\right)$ называют [3] свободной әнергией системы $N$ частищ в объеме $L^{3}$ при температуре $\theta$, асимптотика свободной энергии в термодинамическом пределе имеет вид $N f(\theta, n)+o(N)$. Для идеального газа с учетом (4) из (5) получается, что главный член асимптотики свободной энергии имеет вид

$$
-N \theta \ln \left(\frac{e}{n(2 \pi)^{3}} \int d q e^{-\hbar^{2} q^{2} /(2 m \theta)}\right)=-N \theta \ln \left(\frac{e}{n(2 \pi)^{3}}\left(\frac{2 m \pi \theta}{\hbar^{2}}\right)^{3 / 2}\right)
$$

где $e$ - основание натурального логарифма, $q \in \mathbb{R}^{3}$.

Покажем теперь на примере идеального газа, что асимптотика выражения (5) совпадает с асимптотикой минимального собственного значения некоторого оператора. Рассмотрим статистический ансамбль [3] из $K$ одинаковых систем $N$ частищ на торе $\mathbb{T}$ с гамильтонианом (1) и будем считать, что эти $K$ систем образуют одну систему: $K N$ частищ на торе $\widetilde{\mathbb{T}}$, все три окружности которого равны $\sqrt[3]{K} L$, с гамильтонианом $\widehat{\widetilde{H}}_{K N}$ вида $(1)$, действующим в пространстве $L_{2}\left(\widetilde{T}^{K N}\right)$. Из принципа термодинамической аддитивности [3] следует, что в термодинамическом пределе для любого значения $K$ имеет место асимптотическое равенство

$$
-\frac{\theta}{N} \ln \left(\frac{1}{N !} \operatorname{Sp} e^{-\widehat{H}_{N} / \theta}\right) \sim-\frac{\theta}{(K N) !} \ln \left(\operatorname{Sp} e^{-\widehat{\widetilde{H}}_{K N} / \theta}\right) .
$$

Формула (6) в случае идеального газа позволяет убедиться в этом непосредственно. Рассмотрим $\operatorname{Sp} e^{-\widehat{\widetilde{H}}_{K N} / \theta} ;$ в силу того, что $\widehat{\widetilde{H}}_{K N}$ имеет вид (1), только переменная $x$ лежит на большом торе $\widetilde{\mathbb{T}}$, собственные значения этого оператора имеют вид, аналогичньй (4), и могут быть записаны в виде

$$
\widetilde{E}_{\{p\}}=\sum_{j=1}^{N} \sum_{l=1}^{K} \frac{\hbar^{2} p_{j l}^{2}}{2 m}
$$

где $p_{j l}$ - трехмерные векторы вида $\frac{2 \pi}{L \sqrt[3]{K}}\left(K_{1}, K_{2}, K_{3}\right), K_{1}, K_{2}, K_{3}$ - целые числа. Поэтому далее

$$
\operatorname{Sp} e^{-\widehat{\widetilde{H}}_{K N} / \theta}=\left(\sum_{p_{1}} \sum_{p_{2}} \cdots \sum_{p_{N}} e^{-\hbar^{2} /(2 m \theta) \sum_{j=1}^{N} p_{j}^{2}}\right)^{K} .
$$


Используя метод, аналогичньй развитым в [1], [2], можно показать, что в термодинамическом пределе главный член асимптотики правой части равенства (9) совпадает с главным членом асимптотики выражения

$$
K ! K^{N} \sum_{\{N K\}} \prod_{\{K\}}\left(e^{-\frac{E_{\{K\}}}{\theta} N_{\{K\}}} e^{N_{\{K\}}} e^{-N_{\{K\}} \ln N_{\{K\}}}\right),
$$

где $E_{\{K\}}$ имеет вид (4), $\{K\}$ - введенные выше наборы векторов $K_{1}, \ldots, K_{N}, \prod_{\{K\}}-$ произведение по всем таким наборам, $N_{\{K\}}$ - целое число, зависящее от $\{K\},\{N\}-$ обозначение для набора всех таких чисел, $\sum_{\{N\}, K}-$ обозначение для суммы по всем наборам $\{N\}$ таким, что $\sum_{\{K\}} N_{\{K\}}=K$. Очевидно, что выражение (19) совпадает с выражением

$$
K ! K^{N} \operatorname{Sp}_{K}\left(e^{-K \widehat{A} / \theta}\right),
$$

где $\widehat{A}$ - вторично квантованньй оператор в бозонном фоковском пространстве [4]:

$$
\begin{aligned}
\widehat{A}= & \sum_{\{K\}}\left(E_{\{K\}} \frac{\widehat{N}\left(K_{1}, \ldots, K_{N}\right)}{K}-\theta \frac{\widehat{N}\left(K_{1}, \ldots, K_{N}\right)}{K}\right. \\
& \left.+\theta \frac{\widehat{N}\left(K_{1}, \ldots, K_{N}\right)}{K} \ln \widehat{N}\left(K_{1}, \ldots, K_{N}\right)\right),
\end{aligned}
$$

где

$$
\widehat{N}\left(K_{1}, \ldots, K_{N}\right)=\widehat{\psi}^{+}\left(K_{1}, \ldots, K_{N}\right) \widehat{\psi}^{-}\left(K_{1}, \ldots, K_{N}\right),
$$

$\widehat{\psi}^{+}\left(K_{1}, \ldots, K_{N}\right)$ и $\widehat{\psi}^{-}\left(K_{1}, \ldots, K_{N}\right)$ - операторы рождения и уничтожения [4] в фоковском пространстве, $\mathrm{Sp}_{K}$ означает, что берется след ограниченного оператора на $K$-частичное подпространство пространства Фока. Все асимптотические равенства, написанные до сих пор, справедлливы при любом $K$, поэтому мы можем рассмотреть предел $K \rightarrow \infty$.

Легко убедиться, аналогично тому, как это было сделано в [1], [2], что в этом пределе асимптотика $\operatorname{Sp}_{K}\left(e^{-K \widehat{A} / \theta}\right)$ в главном члене совпадает с $e^{-K a / \theta}$, где $a-$ минимальное собственное значение оператора $\widehat{A}$ на $K$-частичном подпространстве. Из этого с учетом (7) и (9) следует, что свободная энергия идеального газа из $N$ частиц на торе $\mathbb{T}$ с гамильтонианом (1) при температуре $\theta$ в термодинамическом пределе асимптотически эквивалентна минимальному собственному значению вторично квантованного операто$\mathrm{pa}$

$$
\begin{aligned}
\widehat{F}_{\theta, K}= & \sum_{\{K\}} E_{\{E\}} \frac{\widehat{N}\left(K_{1}, \ldots, K_{N}\right)}{K} \\
& +\theta \sum_{\{K\}} \frac{\widehat{N}\left(K_{1}, \ldots, K_{N}\right)}{K} \ln \left(\frac{\widehat{N}\left(K_{1}, \ldots, K_{N}\right)}{K}\right)-\theta+\theta N \ln N
\end{aligned}
$$

на $K$-частичном подпространстве при $K \rightarrow \infty$. Оператор (12) отличается от (11) на число, не зависящее от операторов $\widehat{\psi}^{ \pm}\left(K_{1}, \ldots, K_{N}\right)$. Этот оператор является аналогом операторов свободной энергии, полученных в [1], [2]. При операторах $\widehat{\psi}^{ \pm}\left(K_{1}, \ldots, K_{N}\right)$ 
стоит мальй параметр $1 / \sqrt{K}$, это позволяет строить асимптотику собственных значений оператора (12) методами, развитыми в [5]. Согласно [5] главньй член асимптотики при $K \rightarrow \infty$ собственных значений оператора (12) совпадает с собственньми значениями следующей системы уравнений:

$$
\begin{gathered}
\lambda \widetilde{\Psi}\left(K_{1}, \ldots, K_{N}\right)=\sum_{j=1}^{N} \frac{\hbar^{2} K_{j}^{2}}{2 m} \widetilde{\Psi}\left(K_{1}, \ldots, K_{N}\right)+\theta \ln \left(\left|\widetilde{\Psi}\left(K_{1}, \ldots, K_{N}\right)\right|^{2}\right) \widetilde{\Psi}\left(K_{1}, \ldots, K_{N}\right), \\
\sum_{K_{1}} \cdots \sum_{K_{N}}\left|\widetilde{\Psi}\left(K_{1}, \ldots, K_{N}\right)\right|^{2}=1 .
\end{gathered}
$$

Перейдем в $x$-представление. Пусть $\{K\}_{\alpha}$ - некоторое упорядочивание наборов $\{K\}$, т.е. взаимно однозначное отображение натуральных чисел $\alpha=1,2, \ldots$ и множества наборов векторов $K_{1}, \ldots, K_{N}$. Введем функции

$$
\Psi_{\alpha}\left(x_{1}, \ldots, x_{N}\right)=\Psi_{\{K\}_{\alpha}}\left(x_{1}, \ldots, x_{N}\right)\left|\widetilde{\Psi}\left(K_{1 \alpha}, \ldots, K_{N \alpha}\right)\right|
$$

где $\Psi_{\{K\}}\left(x_{1}, \ldots, x_{N}\right)$ - функции (3). Очевидно, что функции (14) удовлетворяют системе уравнений

$$
\begin{gathered}
\lambda \Psi_{\alpha}\left(x_{1}, \ldots, x_{N}\right)=\widehat{H}_{N} \Psi_{\alpha}\left(x_{1}, \ldots, x_{N}\right)+\theta \ln \left(\left\|\Psi_{\alpha}\right\|^{2}\right) \Psi_{\alpha}\left(x_{1}, \ldots, x_{N}\right), \\
\sum_{\alpha=1}^{\infty}\left\|\Psi_{\alpha}\right\|^{2}=1, \quad\left(\Psi_{\alpha}, \Psi_{\beta}\right)=0 \quad \text { при } \alpha \neq \beta,
\end{gathered}
$$

где $\left\|\Psi_{\alpha}\right\|$ - норма функции $\Psi_{\alpha}\left(x_{1}, \ldots, x_{N}\right)$ в $L_{2}\left(\mathbb{T}^{N}\right)$, а $\widehat{H}_{N}$ имеет вид (1). Будем теперь считать, что $\widehat{H}_{N}$ - произвольный гамильтониан и нам известны его собственные значения и собственные функции $\mathscr{E}_{n}$, и $\Phi_{n}\left(x_{1}, \ldots, x_{M}\right), n=1,2, \ldots$, образуют полную ортонормированную систему в $L_{2}\left(\mathbb{T}^{N}\right)$. В таком случае все решения системы $(15)$ могут быть предъявлены. Например, уравнения (15) имеют решения вида $\Psi_{\alpha}\left(x_{1}, \ldots, x_{N}\right)=$ $\delta_{\alpha n} \Phi_{n}\left(x_{1}, \ldots, x_{N}\right), \lambda=\mathscr{E}_{n}$ (мы учитьваем, что $a \ln a=0$ при $\left.a=0\right)$. Легко также убедиться, что существуют решения вида

$$
\begin{aligned}
\Psi_{\alpha}\left(x_{1}, \ldots, x_{N}\right) & =a_{1} \delta_{\alpha n_{1}} \Phi_{n 1}\left(x_{1}, \ldots, x_{N}\right)+a_{2} \delta_{\alpha n_{2}} \Phi_{n 1}\left(x_{1}, \ldots, x_{N}\right), \\
\lambda & =-\theta \ln \left(e^{-\mathscr{E}_{n_{1}} / \theta}+e^{-\mathscr{E}_{n_{2}} / \theta}\right),
\end{aligned}
$$

где

$$
a_{1,2}=\sqrt{\frac{e^{-\mathscr{E}_{n_{1}, n_{2}} / \theta}}{e^{-\mathscr{E}_{n_{1}} / \theta}+e^{-\mathscr{E}_{n_{2}} / \theta}}} .
$$

Вообще, для любого $l \geqslant 1$ функции

$$
\Psi_{\alpha}\left(x_{1}, \ldots, x_{N}\right)=\sum_{s=1}^{l} a_{s} \delta_{\alpha n_{s}} \Phi_{n_{s}}\left(x_{1}, \ldots, x_{N}\right),
$$

где $n_{s}$ - произвольный набор чисел $n_{1} \neq n_{2} \neq \cdots \neq n_{l}$, являются решениями системы (15) с собственным значением

$$
\lambda=-\theta \ln \left(\sum_{s=1}^{l} e^{-\mathscr{E}_{n_{s}} / \theta}\right)
$$


при условии, что

$$
a_{s}=\sqrt{e^{-\mathscr{E}_{n_{s}} / \theta} / \sum_{r=1}^{l} e^{-\mathscr{E}_{n_{r}} / \theta}}
$$

Формулы (16) и (17) распространяются и на случай бесконечного числа $l$. А именно, имеет место

УТВЕРЖДЕНИЕ. Набор функиий $\Psi_{\alpha}\left(x_{1}, \ldots, x_{N}\right)$ является решением системь уравнений (15) тогда и только тогда, когда этот набор имеет вид

$$
\Psi_{\alpha}\left(x_{1}, \ldots, x_{N}\right)=a_{\alpha} \Phi_{n_{\alpha}}\left(x_{1}, \ldots, x_{N}\right) b_{\alpha}, \quad \alpha=1,2, \ldots,
$$

әде $n_{\alpha}$ - взаимно однозначное отображсние множества натуральных чисел на себя, $b_{\alpha}-$ коэффиииенты, принимающие значения $0 u 1, a_{\alpha}$ имеют вид

$$
a_{\alpha}=\sqrt{e^{-\mathscr{E}_{n_{\alpha}} / \theta} / \sum_{\beta=1}^{\infty} b_{\beta} e^{-\mathscr{E}_{n_{\beta}} / \theta}}
$$

Соответствующее собственное значение есть

$$
\lambda=-\theta \ln \left(\sum_{\alpha=1}^{\infty} b_{\alpha} e^{-\mathscr{E}_{n_{\alpha}} / \theta}\right) .
$$

Исследуем множество собственных значений (18). Так как $b_{\alpha}=0$ или $1, \theta>0$, то выражение (18) тем меньше, чем больше в сумме слагаемьх, отличньх от 0. Очевидно, что минимальное из всех значений (18) есть

$$
\lambda=-\theta \ln \left(\sum_{n=1}^{\infty} e^{-\mathscr{E}_{n} / \theta}\right)
$$

и совпадает со свободной энергией системы $N$ частиц на торе $\mathbb{T}$ с гамильтонианом $\widehat{H}_{N}$ при температуре $\theta$ [3]. Будем считать, что $\mathscr{E}_{n} \leqslant \mathscr{E}_{n+1}$. В этом случае ближайшим к самому нижнему значению $\lambda$ будет $-\theta \ln \left(\sum_{n=2}^{\infty} e^{-\mathscr{E}_{n} / \theta}\right)$, и т.д. Как было показано, среди собственных значений (18) содержатся все собственные значения $\mathscr{E}_{n}$ гамильтониана $\widehat{H}_{N}$. Вообще, все собственные значения (18) разбиваются на классы следующим образом: при $\theta \rightarrow 0$ выражение $(18)$ в зависимости от $b_{\alpha}$ сходится к какому-либо значению $\mathscr{E}_{n}, \lambda$, имеюшие одинаковьй предел при $\theta \rightarrow 0$, относятся к одному классу.

Итак, для произвольного гамильтониана $\widehat{H}_{N}$ было показано, что минимальное собственное значение системы уравнений (15) совпадает со свободной энергией при температуре $\theta$. Заметим, что то же самое справедливо в случае, когда рассматриваются бозоны или фермионы, т.е. система $(15)$ и гамильтониан $\widehat{H}_{N}$ рассматриваются только на симметричных или антисимметричных функциях. Таким образом, для случая идеального газа показано, что асимптотика свободной энергии совпадает с асимптотикой минимального собственного значения оператора (12). Естественно поэтому далее квантовать свободную энергию идеального газа следующим образом: собственньги значениями свободной энергии идеального газа будем называть предел собственных значений оператора $(12)$ при $K \rightarrow \infty$. Как было уже показано, этот предел совпадает 
с собственньми значениями $\lambda$ системы уравений (13). Ниже предъявляется оператор аналогичньй $(12)$ в случае, когда $\widehat{H}_{N}$ произвольньй, и статистический ансамбль ультравторично квантуется.

Рассмотрим теперь ультравторично квантованную систему $N$ бозонов или фермионов на торе $\mathbb{T}$, которые образуют $K$ пар “частища и статистический спин” и $M$ пар "частища и частища" [1], [2], $K+2 M=N$. Состояние такой системы описьвается функцией $\Phi_{K, M}\left(x_{1}, s_{1} ; \ldots ; x_{K}, s_{K} ; y_{1}, 2 ; \ldots ; y_{2 M-1}, y_{2 M}\right)$, которая симметрична относительно перестановок пар переменньх $x_{i}, s_{i}$ и $x_{j}, s_{j}$ и симметрична относительно перестановок пар переменных $\left(y_{2 \alpha-1}, y_{2 \alpha}\right)$ и $\left(y_{2 \beta-1}, y_{2 \beta}\right)$. Кроме того, в бозонном случае $\Phi_{K, M}$ - симметричная, а в фермионном - антисимметричная функция $N$ переменных $x_{1}, x_{2}, \ldots, x_{K}, y_{1}, \ldots, y_{2}$ [1], [2]. Будем считать, что гамильтониан системы имеет вид

$$
\widehat{H}_{N}=-\frac{\hbar^{2}}{2 m} \sum_{j=1}^{N} \Delta_{j}+\sum_{j=1}^{N} \sum_{l=j+1}^{N} V\left(x_{j}-x_{l}\right),
$$

хотя все дальнейшие рассуждения справедливы и в случае гамильтонианов более общего вида. Рассмотрим ансамбль из $Z$ таких систем. Аналог оператора (12) в этом случае имеет вид

$$
\begin{aligned}
\widehat{F}_{\theta, Z}= & \sum_{s_{1}=0}^{\infty} \cdots \sum_{s_{K}=0}^{\infty} \frac{1}{Z} \int \cdots \int d x_{1} \cdots d x_{K+2 M} \\
& \times \widehat{\psi}^{+}\left(x_{1}, s_{1} ; x_{2}, s_{2} ; \ldots ; x_{K}, s_{K} ; x_{K+1}, x_{K+2} ; \ldots ; x_{K+2 M-1}, x_{K+2 M}\right) \widehat{H}_{K+2 M} \\
& \times \widehat{\psi}^{-}\left(x_{1}, s_{1} ; x_{2}, s_{2} ; \ldots ; x_{K}, s_{K} ; x_{K+1}, x_{K+2} ; \ldots ; x_{K+2 M-1}, x_{K+2 M}\right) \\
& +\theta \int \cdots \int d x_{1} d y_{1} \cdots d x_{K+2 M} d y_{K+2 M} \\
& \times r\left(x_{1}, \ldots, x_{K+2 M} ; y_{1}, \ldots, y_{K+2 M}\right)(\ln r)\left(y_{1}, \ldots, y_{K+2 M} ; x_{1}, \ldots, x_{K+2 M}\right) \\
& -\theta+\theta(K+2 M) \ln (K+2 M)
\end{aligned}
$$

где $\widehat{\psi}^{ \pm}\left(x_{1}, s_{1} ; \ldots ; x_{K}, s_{K} ; y_{1}, y_{2} ; \ldots ; y_{2 M-1}, y_{2 M}\right)$ - бозонные операторы рождения и уничтожения,

$$
\begin{aligned}
r\left(x_{1}, \ldots, x_{K+2 M} ; y_{1}, \ldots, y_{K+2 M}\right) \\
=\frac{1}{Z} \sum_{s_{1}=0}^{\infty} \cdots \sum_{s_{K}=0}^{\infty} \widehat{\psi}^{+}\left(x_{1}, s_{1} ; \ldots ; x_{K}, s_{K} ; x_{K+1}, x_{K+2} ; \ldots ; x_{K+2 M-1}, x_{K+2 M}\right) \\
\quad \times \widehat{\psi}^{-}\left(y_{1}, s_{1} ; \ldots ; y_{K}, s_{K} ; y_{K+1}, y_{K+2} ; \ldots ; x_{K+2 M-1}, x_{K+2 M}\right) .
\end{aligned}
$$

Выражение (20) можно рассматривать как операторнозначное ядро оператора $\widehat{r}$ в $L_{2}\left(\mathbb{T}^{K+2 M}\right),(\ln r)\left(x_{1}, \ldots, x_{K+2 M} ; y_{1}, \ldots, y_{K+2 M}\right)$ - обозначение для операторнозначного ядра оператора $\ln \widehat{r}_{\text {в }} L_{2}\left(\mathbb{T}^{K+2 M}\right)$. Собственньми значениями свободной энергии ультравторично квантованной системы при температуре $\theta$ будем назьвать предел собственных значений оператора (20) на $Z$-частичном подпространстве при $Z \rightarrow \infty$. Поскольку при операторах $\widehat{\psi}^{ \pm}$в операторе $\widehat{F}_{\theta, Z}$ стоит мальй параметр $1 / \sqrt{Z}$, то для нахождения предела собственных значений этого оператора при $Z \rightarrow \infty$ применимы методы, развитые в [5]. Согласно [5] этот предел совпадает с собственньци значениями $\lambda$ 
следуюшей системы уравнений:

$$
\begin{aligned}
& \left(\widehat{H}_{K+2 M}+\theta \ln \widehat{\rho}\right) \Psi\left(x_{1}, s_{1} ; \ldots ; x_{K}, s_{K} ; y_{1}, y_{2} ; \ldots ; y_{2 M-1}, y_{2 M}\right) \\
& \quad=\lambda \Psi\left(x_{1}, s_{1} ; \ldots ; x_{K}, s_{K} ; y_{1}, y_{2} ; \ldots ; y_{2 M-1}, y_{2 M}\right), \quad \operatorname{Sp} \hat{\rho}=1
\end{aligned}
$$

где $\widehat{\rho}$ - оператор в пространстве $L_{2}\left(\mathbb{T}^{K+2 M}\right)$, ядро которого имеет вид

$$
\begin{aligned}
& \rho\left(x_{1}, \ldots, x_{K+2 M} ; y_{1}, \ldots, y_{2 M+K}\right) \\
& \quad=\sum_{s_{1}=0}^{\infty} \cdots \sum_{s_{K}=0}^{\infty} \Psi\left(x_{1}, s_{1} ; \ldots ; x_{K}, s_{K} ; x_{K+1}, x_{K+2} ; \ldots ; x_{K+2 M-1}, x_{K+2 M}\right) \\
& \quad \times \Psi^{*}\left(y_{1}, s_{1} ; \ldots ; y_{K}, s_{K} ; y_{K+1}, y_{K+2} ; \ldots ; y_{K+2 M-1}, y_{K+2 M}\right) .
\end{aligned}
$$

Система (22) согласно [1] является системой уравнений с унитарной нелинейностью, так как умножение решения $\Psi$ на множитель $e^{i \varphi}, \varphi \in \mathbb{R}$, также дает решение (22) с тем же самым значением $\lambda$. Методы нахождения решений таких уравнений развиты в [1]. Запишем систему (22) в виде одного ультравторично квантованного уравнения.

В [1], [2] было введено пространство ультравторичного квантования $\mathscr{F}$, которое является частным случаем пространства Фока. Операторы рождения и уничтожения в $\mathscr{F}$ обозначаются $\widehat{b}^{+}(x, s), \widehat{B}^{+}\left(y_{1}, y_{2}\right)$ и $\widehat{b}^{-}(x, s), \widehat{B}^{-}\left(y_{1}, y_{2}\right)$. Кроме того, рассматриваются "обычное" бозонное или фермионное фоковское пространство $\mathscr{H}_{B}$ или $\mathscr{H}_{F}$, операторы рождения и уничтожения в этом пространстве обозначаются через $\widehat{\psi}^{+}(x)$ и $\widehat{\psi}^{-}(x)$. Каждому вектору $\Phi \in \mathscr{F}$ сопоставим вторично квантованньй оператор $\widehat{R}(\Phi)$ в пространстве $\mathscr{H}_{B, F}$ :

$$
\begin{aligned}
\widehat{R}(\Phi)= & \left\{\sum_{K=0}^{\infty} \sum_{M=0}^{\infty} \frac{1}{K ! M !(K+2 M) !}\right. \\
& \times \sum_{s_{1}=0}^{\infty} \cdots \sum_{s_{K}=0}^{\infty} \int \cdots \int d x_{1} d x_{1}^{\prime} \cdots d x_{K} d x_{K}^{\prime} d y_{1} d y_{1}^{\prime} \cdots d y_{2 M} d y_{2 M}^{\prime} \\
& \times \widehat{\psi}^{+}\left(y_{1}\right) \cdots \widehat{\psi}^{+}\left(y_{2 M}\right) \widehat{\psi}^{+}\left(x_{1}\right) \cdots \widehat{\psi}^{+}\left(x_{K}\right) \\
& \times \widehat{\mathscr{P}}_{0 B, F} \widehat{\psi}^{-}\left(x_{K}^{\prime}\right) \cdots \widehat{\psi}^{-}\left(x_{1}^{\prime}\right) \widehat{\psi}^{-}\left(y_{2 M}^{\prime}\right) \cdots \widehat{\psi}^{-}\left(y_{1}^{\prime}\right) \\
& \times\left(\Phi, \widehat{b}^{+}\left(x_{1}, s_{1}\right) \cdots \widehat{b}^{+}\left(x_{K}, s_{K}\right) \widehat{B}^{+}\left(y_{1}, y_{2}\right) \cdots \widehat{B}^{+}\left(y_{2 M-1}, y_{2 M}\right)\right. \\
& \times \exp \left(-\sum_{s=0}^{\infty} \int d x \widehat{b}^{+}(x, s) \widehat{b}^{-}(x, s)-\iint d y d y^{\prime} \widehat{B}^{+}\left(y, y^{\prime}\right) \widehat{B}^{-}\left(y, y^{\prime}\right)\right) \\
& \times \widehat{B}^{-}\left(y_{2 M-1}^{\prime}, y_{2 M}^{\prime}\right) \cdots \widehat{B}^{-}\left(y_{1}^{\prime}, y_{2}^{\prime}\right) \widehat{b}^{-}\left(x_{K}^{\prime}, s_{K}\right) \cdots \\
& \left.\left.\times \widehat{b}^{-}\left(x_{1}^{\prime}, s_{1}\right) \Phi\right)\right\} /\left(\Phi, \widehat{E}_{B, F} \Phi\right),
\end{aligned}
$$

где $\overline{\widehat{E}}_{B, F}$ - введенные в [1], [2] ультравторично квантованные единичные операторы, $\widehat{\mathscr{P}}_{0 B, F}$ - оператор ортогонального проектирования на вакуумньй вектор пространства $\mathscr{H}_{B, F}$, числа над операторами обозначают порядок их действия. Набору операторов 
$\widehat{H}_{N}, N=0,1, \ldots$, вида (19) в работах [1], [2] сопоставлен ультравторично квантованный оператор $\overline{\widehat{H}}$. Кроме того, в этих работах предъявлен способ, с помощью которого любому вторично квантованному оператору сопоставляется ультравторично квантованньй оператор. Будем обозначать через $\overline{\widehat{L}}(\Phi)$ ультравторично квантованньй оператор, который согласно [1], [2] отвечает вторично квантованному оператору $\ln \widehat{R}(\Phi)$. Имеет место

Teоpema 1. Пусть вектор $\Phi \in \mathscr{F}$ имеет вид

$$
\begin{aligned}
\Phi=\frac{1}{\sqrt{K ! M !}} \sum_{s_{1}=0}^{\infty} \cdots \sum_{s_{K}=0}^{\infty} \int \cdots \int d x_{1} \cdots d x_{K+2 M} \\
\quad \times \Psi\left(x_{1}, s_{1} ; \ldots ; x_{K}, s_{K} ; x_{K+1}, x_{K+2} ; \ldots ; x_{K+2 M-1}, x_{K+2 M}\right) \\
\quad \times \widehat{b}^{+}\left(x_{1}, s_{1}\right) \cdots \widehat{b}^{+}\left(x_{K}, s_{K}\right) \widehat{B}^{+}\left(x_{K+1}, x_{K+2}\right) \cdots \widehat{B}^{+}\left(x_{K+2 M-1}, x_{K+2 M}\right) \Phi_{0},
\end{aligned}
$$

где функиия $\Psi\left(x_{1}, s_{1} ; \ldots ; x_{K}, s_{K} ; x_{K+1}, x_{K+2} ; \ldots ; x_{K+2 M-1}, x_{K+2 M}\right)$ удовлетворяет системе (22). Тогда $\Phi$ удовлетворяет уравнению

$$
(\overline{\widehat{H}}+\theta \overline{\widehat{L}}(\Phi)) \Phi=\lambda \overline{\widehat{E}} \Phi
$$

ДокАЗАТЕЛЬСТво проводится непосредственно подстановкой вектора (25) в уравнение (26).

Рассмотрим на пространстве $\mathscr{F}$ функционал

$$
\mathscr{E}=\frac{(\Phi, \overline{\widehat{H}} \Phi)}{(\Phi, \overline{\widehat{E}} \Phi)}
$$

и будем искать экстремум данного функционала при дополнительных условиях

$$
\overline{\widehat{N}} \Phi=N \overline{\widehat{E}} \Phi, \quad S=-\operatorname{Sp}(\widehat{R}(\Phi) \ln \widehat{R}(\Phi))
$$

где $\overline{\widehat{N}}$ - ультравторично квантованный оператор числа частиц [1], [2], $N, S$ - фиксированные число частиц и энтропия системы. Уравнение экстремума функционала (27) при условиях (28) может быть приведено к виду (26), температура $\theta$ появляется как множитель Лагранжа при условии $S=$ const, а экстремальное значение (27) есть $\varepsilon=\lambda+\theta S$. Таким образом, возможно проквантовать термодинамику еще одним эквивалентным способом: собственными значениями внутренней энергии системы при заданной энтропии $S$ и числа частиц $N$ будем назьвать экстремальные значения функционала (27) при условиях (28).

Теорема 2. Минимальное собственное значение внутренней энергии в термодинамическом пределе асимптотически совпадает с термодинамической внутренней энергией системы. 
ДокАЗАТЕЛЬСТво. Минимальное собственное значение $\lambda$ уравнения (26) совпадает со свободной энергией $F=-\theta \ln \left(\mathrm{Sp} e^{-\widehat{H}_{N} / \theta}\right)$. В этом легко убедиться для эквивалентной системы $(22)$ так же, как это было сделано для аналогичной системы уравнений (15). Умножая (26) на $\Phi$, получим

$$
\mathscr{E}=\frac{(\Phi, \overline{\widehat{H}} \Phi)}{(\Phi, \overline{\widehat{E}} \Phi)}=F-\theta \frac{(\Phi, \overline{\widehat{L}}(\Phi) \Phi)}{(\Phi, \widehat{E} \Phi)}
$$

Используя методы, развитые в [1], [2], можно показать, что

$$
\frac{(\Phi, \widehat{\widehat{L}}(\Phi) \Phi)}{(\Phi, \widehat{E} \Phi)}=-\operatorname{Sp}(\widehat{R}(\Phi) \ln \widehat{R}(\Phi))=S
$$

Поэтому из (29) для минимального собственного значения внутренней энтропии при фиксированной энтропии $S$ получаем выражение $F-\theta S$, а это и есть термодинамическая внутренняя энергия.

В заключение отметим, что введенный способ квантования внутренней энергии удобен для построения асимптотик. Например, в пределе, когда коммутаторы между операторами $\widehat{b}^{-}(x, s)$ и $\widehat{b}^{+}\left(x^{\prime}, s^{\prime}\right), \widehat{B}^{-}\left(y_{1}, y_{2}\right)$ и $\widehat{B}^{-}\left(y_{1}^{\prime}, y_{2}^{\prime}\right)$ малы, в функционалах $(27)$ и $(28)$ везде можно заменить операторы $\widehat{b}^{ \pm}$и $\widehat{B}^{ \pm}$на их символы. Минимум полученного таким образом символа внутренней энергии $\mathscr{E}$ при фиксированньх значениях символов числа частиц и энтропии совпадают с полученным с помощью вариационного принципа в теории БКШ выражением для внутренней энергии.

\section{СПИСОК ЦИТИРОВАННОЙ ЛИТЕРАТУРЫ}

[1] Маслов В.П. Квантование термодинамики и ультравторичное квантование. НИЦ "Регулярная и хаотическая динамика". Ижевск: УРСС, 2001.

[2] Маслов В.П. О некоторых тождествах для проквантованной энтропии // ТМФ. 2001. Т. 129. № 3. C. 464-490.

[3] Ландау Л. Д., Лифшиц Е. М. Статистическая физика. М.: Наука, 1976.

[4] Березин Ф. А. Метод вторичного квантования. М.: Наука, 1986.

[5] Маслов В.П., Шведов О. Ю. Метод комплексного ростка. М.: УРСС, 2000.

Московский государственный университет им. М.В. Ломоносова

Поступило 14.02 .2002 